Subscriber access provided by Bethel University

Article

\title{
Conformational isomerization involving conserved proline residues modulates oligomerization of the NS1 interferon response inhibitor from the syncytial respiratory virus
}

Julieta Conci, Damian Alvarez-Paggi, Guilherme A. P. de Oliveira, Talita Duarte

Pagani, Sebastián Andrés Esperante, Silvia Susana Borkosky, Martin Aran, Leonardo Gabriel Alonso, Ronaldo Mohana-Borges, and Gonzalo de Prat-Gay

Biochemistry, Just Accepted Manuscript • DOI: 10.1021/acs.biochem.8b01288 • Publication Date (Web): 06 Jun 2019

Downloaded from http://pubs.acs.org on June 7, 2019

\section{Just Accepted}

"Just Accepted" manuscripts have been peer-reviewed and accepted for publication. They are posted online prior to technical editing, formatting for publication and author proofing. The American Chemical Society provides "Just Accepted" as a service to the research community to expedite the dissemination of scientific material as soon as possible after acceptance. "Just Accepted" manuscripts appear in full in PDF format accompanied by an HTML abstract. "Just Accepted" manuscripts have been fully peer reviewed, but should not be considered the official version of record. They are citable by the Digital Object Identifier (DOI®). "Just Accepted" is an optional service offered to authors. Therefore, the "Just Accepted" Web site may not include all articles that will be published in the journal. After a manuscript is technically edited and formatted, it will be removed from the "Just Accepted" Web site and published as an ASAP article. Note that technical editing may introduce minor changes to the manuscript text and/or graphics which could affect content, and all legal disclaimers and ethical guidelines that apply to the journal pertain. ACS cannot be held responsible for errors or consequences arising from the use of information contained in these "Just Accepted" manuscripts. 


\title{
Conformational isomerization involving conserved proline residues modulates oligomerization of the NS1 interferon response inhibitor from the syncytial respiratory virus
}

\author{
Julieta Conci', Damian Alvarez-Paggi', Guilherme A. P. de Oliveira², Talita D. Pagani, \\ Sebastian A. Esperante1, Silvina S. Borkosky¹, Martin Aran¹, Leonardo G. Alonso', Ronaldo \\ Mohana-Borges ${ }^{3}$, Gonzalo de Prat- Gay ${ }^{1 *}$
}

1 Protein Structure-Function and Engineering Laboratory, Fundación Instituto Leloir and IIBBA-CONICET, Av. Patricias Argentinas 435, 1405 Buenos Aires, Argentina.

2 Programa de Biologia Estrutural, Instituto de Bioquímica Médica Leopoldo de Meis, Instituto Nacional de Ciência e Tecnologia de Biologia Estrutural e Bioimagem, Centro Nacional de Ressonância Magnética Nuclear Jiri Jonas, Universidade Federal do Rio de Janeiro, Rio de Janeiro, Brazil.

3 Instituto de Biofísica Carlos Chagas Filho, Universidade Federal do Rio de Janeiro, Rio de Janeiro, Brazil 
KEYWORDS: Respiratory Syncytial Virus, NS1, interferon, oligomerization, proline 


\section{ABSTRACT}

The interferon response suppression by the respiratory syncytial virus relies on two unique non-structural proteins, NS1 and NS2, that interact with cellular partners through high order complexes. We hypothesized that two conserved proline residues, P81 and P67, participate in the conformational change leading to oligomerization. We found that molecular dynamics of NS1 show a highly mobile C-terminal helix, which become rigid upon in silico replacement of P81. A soluble oligomerization pathway into regular spherical structures at low ionic strength competes with an aggregation pathway at high ionic strength upon temperature increase. P81A requires higher temperatures to oligomerize and has a small positive effect on aggregation, while P67A is largely prone to aggregation. Chemical denaturation shows a first transition, involving a large fluorescence and ellipticity change corresponding to both a conformational change and substantial effects on the environment of its single tryptophan, that is strongly destabilized in P67A but stabilized by P81A. The subsequent global cooperative unfolding corresponding to the main $ß$-sheet core is not affected by the proline mutations. Thus, a clear link exists between the effect of P81 and P67 on the stability of the first transition and oligomerization/aggregation. Interestingly, both P67 and P81 are located 
far away in space and sequence from the C-terminal helix, indicating a marked global structural dynamics. This provide a mechanism for modulating the oligomerization of NS1 by unfolding of a weak helix that exposes hydrophobic surfaces, linked to the participation of NS1 in multiprotein complexes. 


\section{INTRODUCTION}

RNA viruses require the innate immune response to be suppressed in order to attain a productive life cycle. Negative-sense single-stranded RNA viruses (Mononegavirales) use several different strategies mediated by different proteins 1. Paramyxoviruses and Pneumoviruses suppress the IFN response by limiting the synthesis of IFN-inducing viral RNAs, inhibiting RNA sensors, using decoy substrates for cellular kinases, and by direct repressing the IFN promoter ${ }^{2}$. The IFN shut-down in these families of related viruses operate via targeted proteolysis and sequestering of signaling and cellular factors, and upregulation of cellular inhibitors related to IFN induction and IFN effector functions ${ }^{2,3,4}$.

The respiratory syncytial virus (RSV), formerly member of the Paramyxoviridae family and recently reclassified within the new Pneumoviridae family ${ }^{5}$ is responsible for the majority of bronchiolitis and pneumonia cases in infants, elderly and immunocompromised ${ }^{6,7}$. Its 15 kb RNA genome encoding 11 proteins bears two unique 3' proximal genes encoding for two non-structural proteins, NS1 and NS2, that partake in suppressing the innate immune response ${ }^{3}$. These proteins have no sequence homologues among Mononegavirales and are responsible for the interferon (IFN) suppression activity of RSV, a function that is otherwise 
mediated by co-translational editing of the phosphoprotein $\mathrm{P}$ in paramyxoviruses and other pneumoviruses ${ }^{6,7}$. NS1 and NS2 are regarded as multifunctional, they are expressed abundantly immediately after cell entry ${ }^{8}$ and were proposed to form heterodimers ${ }^{9,4}$. In addition, it was shown that NS1 has a strong inhibitory effect on RSV genomic and antigenomic RNA synthesis ${ }^{10}$. NS1 was reported to interact directly or indirectly with 221 targets in a quantitative proteomic analysis ${ }^{11}$ and its multiple functions were recently summarized in Chatterjee et al ${ }^{12}$. The first protein described to co-localize with NS1 was the mitochondrial signaling protein (MAVS), a critical molecule in the type I IFN pathway ${ }^{13}$. NS1 can also bind directly to IRF- 3 and the CREB binding protein (CBP), both transcriptional activators of the type I IFN system ${ }^{14}$.

Recombinant NS1 was shown to be a monomer in solution which unfolds following a highly cooperative two-state transition ${ }^{15,16}$. The native NS1 monomer follows a simple unfolding kinetics with a half-life of $45 \mathrm{~min}$, in agreement with a highly stable core structure at equilibrium ${ }^{17}$. Refolding is a complex process that involves several slowly interconverting species compatible with proline isomerization. However, upon mild temperature variation, conformational changes are induced leading to the formation of regular ca $30 \mathrm{~nm}$ spherical 
oligomers (NS1SOs) which are highly stable and homogeneous ${ }^{16}$. A substantial secondary structure change into increased $\beta$-sheet content and the formation of a dimeric nucleus precede polymerization by the sequential addition of monomers at a surprisingly low rate ${ }^{17}$.

NS1 transfected in lung epithelial cells was shown to form homo-oligomers and to interact with NS2 ${ }^{18}$ suggesting that NS1 oligomerization is a likely event in the cell. In addition, a degradosome consisting of a large heterogeneous protein complex with a molecular mass in the range of $300-750 \mathrm{kDa}$, containing both NS1 and NS2 proteins as well as proteasome subunits, was described ${ }^{19}$. This degradosome also presents degradative activity for NS1 and NS2 cellular targets such as RIG-I, TRAF3, and STAT2 and was found to be important in innate immunity suppression in infected cells ${ }^{19}$. Overall, these findings strongly suggest that the NS1SOs formed quasi-spontaneously in vitro could be linked to the degradosome complex.

The recently determined crystal structure of NS1 showed that the overall fold corresponds to a $\beta$-sandwich composed of seven $\beta$-strands and is completed by three $\alpha$ helices: $\alpha 1$, located between $\beta 2$ and $\beta 3$, the short $\alpha 2$ found right before $\beta 5$, and the $C$-terminal $\alpha 3$ that lies against the $\beta$-sheet core of the protein ${ }^{12}$ ( Figure S1). The multiple binding 
activities of NS1 can take place at a few binding interfaces at most, something to be considered remarkable given its small size $(15 \mathrm{kDa})$. Therefore, conformational heterogeneity and in particular, oligomer formation, may explain such a promiscuous binding activity. Given that oligomerization requires a conformational rearrangement, we hypothesize that oligomerization capacity may be linked to the stability or lack of thereof. We observe two conserved proline residues (P67 and P81) located at secondary structure boundaries (Figure 1 and Figure S1) which, together with the presence of proline type isomerizations in the refolding reaction ${ }^{17}$, made us consider analyzing the role of these residues in conformational stability and oligomerization. In addition, a recent report highlighted the role of prolines as conformational switches in amyloid oligomerization ${ }^{20}$. The fact that NS1 also undergoes a repetitive ß-sheet type oligomerization, reinforced our hypothesis of conserved prolines as modulators of this process.

In this work, we analyze the role of the two conserved proline residues in conformational dynamics and stability, and their participation in oligomerization and aggregation processes using biophysical techniques. We find that the proline mutations display mimimum effect on the global unfolding but affect a first transition correlating chemical stability to oligomerization. 
A novel insoluble aggregation route is uncovered, different and competing with the soluble oligomerization route. Both require a conformational change or local unfolding events that renders aggregation/oligomerization compatible species depending on the ionic strength. We argue that this event involves the separation of the C-terminal $\alpha 3$ helix from the $\beta$-sheet core with a dynamic fast exchanging equilibrium in native conditions. The distant location of the prolines from this a3-helix suggest global dynamics behind the modulation of oligomerization. We discuss the results in the light of reports on the impact on oligomerization on its IFN suppression activity. 


\section{Materials and methods}

\section{Protein Expression and Purification.}

NS1 WT and proline mutants P67A, P81A and P67/81A were expressed and purified as previously described for the human RSV strain A NS1 WT protein ${ }^{16}$. Briefly, C41 cells were transformed with a pMal 2C plasmid, containing NS1 fused with the -maltose binding protein (MBP) with a thrombin cleavage site between the two proteins. Cells were grown in LB medium until an OD of 0.6 was reached and protein expression was induced adding $0.3 \mathrm{mM}$ isopropyl $\beta$-D-1-thiogalactopyranoside and incubating the cells at $37^{\circ} \mathrm{C}$ for $16 \mathrm{~h}$. Cell pellets were resuspended in lysis buffer containing 20 mM Tris. $\mathrm{HCl}(\mathrm{pH} 8.0), 200 \mathrm{mM} \mathrm{NaCl}, 2 \mathrm{mM} 2-$ mercaptoethanol and $1 \mathrm{mM}$ EDTA and lysed by sonication. Supernatants were precipitated with $50 \%$ ammonium sulphate and the pellet was resuspended and dialyzed against a buffer containing $10 \mathrm{mM}$ Tris. $\mathrm{HCl}(\mathrm{pH} 8.0), 200 \mathrm{mM} \mathrm{NaCl}$, and $1 \mathrm{mM}$ DTT. The fusion protein was purified by an affinity amylose resin (New England Biolabs, Hitchin, U.K.), followed by a thrombin cleavage and size exclusion chromatography to separate NS1 from MBP. Highly purified NS1 was dialyzed in $10 \mathrm{mM}$ Tris. $\mathrm{HCl}(\mathrm{pH} 8.0), 100 \mathrm{mM} \mathrm{NaCl}$ and $1 \mathrm{mM}$ DTT. The 
protein concentration was determined spectrophotometrically using a molar extinction coefficient (ع280) of $9970 \mathrm{M}^{-1} \mathrm{~cm}^{-1}$.

\section{Far-UV circular dichroism spectroscopy (CD).}

Far-UV CD measurements were carried out on a Jasco J-810 spectropolarimeter using a Peltier temperature-controlled sample holder at $25{ }^{\circ} \mathrm{C}$ in a $0.1 \mathrm{~cm}$ path length cell. Spectra were recorded between 200 and $260 \mathrm{~nm}$. All the spectra were obtained through an average of six scans. Mean residue molar ellipticity or $[\theta]_{\text {MRW }}$ can be calculated applying the following formula to the raw data:

$$
[\theta]_{M R W}\left(\operatorname{deg} c m^{2} / d m o l\right)=\frac{m \mathrm{deg}}{10 \times L \times[M] \times N}
$$

Where $L$ is the path length (measured in $\mathrm{cm}$ ), $[\mathrm{M}]$ is the concentration (measured in molar units), and $\mathrm{N}$ is the number of peptide bonds (measured as: number of amino acids -1 ).

Thermal denaturation experiments by Far-UV CD spectroscopy. Ellipticity was recorded at $225 \mathrm{~nm}$ with a temperature increase of $4^{\circ} \mathrm{C} / \mathrm{min}$. Acquisition parameters were the following: 4 
$\mathrm{nm}$ bandwidth, $4 \mathrm{~s}$ response time, and $0.2^{\circ} \mathrm{C}$ data pitch. The protein concentration was 10 $\mu \mathrm{M}$. To calculate thermal transition midpoints, the thermal scans were fit to an irreversible denaturation model as was previously described ${ }^{21,22}$.

\section{Fluorescence Spectroscopy.}

Fluorescence experiments were performed in a FluoroMax-4 (Horiba Scientific) spectrofluorometer. Fluorescence emission spectra were recorded at $25^{\circ} \mathrm{C}$ between 310 and $450 \mathrm{~nm}$ with excitation wavelength of $295 \mathrm{~nm}$ using excitation and emission bandwidths of 5 $\mathrm{nm}$. Fluorescence emission data were analyzed by first subtracting the buffer background. The protein concentration was $10 \mu \mathrm{M}$.

\section{Oligomerization Kinetics.}

Oligomerization was analyzed monitoring the changes occurring in the far-UV CD signal at $225 \mathrm{~nm}$. NS1 was added at $10 \mu \mathrm{M}$ to a cuvette containing $10 \mathrm{mM}$ Tris. $\mathrm{HCl}(\mathrm{pH} 8.0)$ and $2 \mathrm{mM}$ 2-mercaptoethanol, equilibrated at $47^{\circ} \mathrm{C}$. The oligomerization reaction was additionally 
measured following the increase in ThT fluorescence in a Jasco FP-6500 spectrofluorometer. It has been previously shown that NS1 Oligomers tightly bind ThT, while the monomeric form shows negligible binding. The ThT fluorescence was measured exciting at $446 \mathrm{~nm}$ a solution containing $20 \mu \mathrm{M}$ ThT and recording the emission intensity at $483 \mathrm{~nm}$ in a temperaturejumping experiment. The data were fitted to a single exponential process as the best aproximation to the complex kinetics.

\section{Unfolding Kinetics.}

Native NS1 from a concentrated stock solution was diluted 10-fold by manual mixing in 10 $\mathrm{mM}$ Tris. $\mathrm{HCl}(\mathrm{pH} 8.0), 100 \mathrm{mM} \mathrm{NaCl}, 1 \mathrm{mM}$ DTT and $5.0 \mathrm{M} \mathrm{Gdm} . \mathrm{HCl}$, to a final protein concentration of $10 \mu \mathrm{M}$. Unfolding kinetics was followed by changes in the CD signal at 220 $\mathrm{nm}$. Unfolding kinetics was also measured monitoring NS1 fluorescence, originated by its single tryptophan residue, with an excitation wavelength of $295 \mathrm{~nm}$ and an emission wavelength of $330 \mathrm{~nm}$. The temperature was kept constant at $25^{\circ} \mathrm{C}$. 


\section{NMR measurements.}

NMR measurements were performed at $25{ }^{\circ} \mathrm{C}$ on a Bruker Avance III spectrometer (Bruker Instruments, Inc., Bellerica, MA, USA) operating at a proton frequency of $600.2 \mathrm{MHz}$. The 1-1 Jump-and-return water suppression pulse sequence was used for 1H 1D NMR experiments ${ }^{23}$. The following experimental parameters were used in all measurements: 256 scans, $1.85 \mathrm{~s}$ relaxation delay, $1.51 \mathrm{~s}$ acquisition time, $18 \mathrm{ppm}$ spectral width, $131 \mu \mathrm{s}$ delay for binomial water suppression and $32 \mathrm{~K}$ acquisition points. The NMR data were zero-filled, Fourier transformed, phase corrected and converted to a Matlab-compatible format for further processing and analysis with NMRPipe ${ }^{24}$. All spectra were submitted to baseline correction and normalization.

\section{Molecular dynamics (MD) simulations.}

System set-up. Initial NS1 WT structure was obtained from the PDB database, i.e. PDB 5VJ2 12. For simulation of monomeric NS1, molecule A of the asymmetric unit from the PDB was used. The P67A and P81A mutants where generated using FoldX ${ }^{25}$. Molecular dynamics (MD) simulations were performed using AMBER16 ${ }^{26}$ and the ff14SB force field was 
employed for all protein variants ${ }^{27}$. The Visual Molecular Dynamics (VMD) software was employed for visualization and image rendering ${ }^{28}$.

Explicit water simulations and periodic boundary conditions. The production simulations for each system were performed immersing each NS1 structure in a TIP3P water box with $20 \AA$ separation between the protein structure and the box edge. In every case, after preliminary rounds of minimization, an initial constant volume MD simulation was performed in order to heat the system to $27^{\circ} \mathrm{C}$. Subsequently, a constant pressure simulation was carried out to equilibrate the system density. The production MDs consisted of $100 \mathrm{~ns}$ simulations for each analyzed variant. Temperature and pressure were kept constant using the Berendsen thermostat and barostat respectively ${ }^{29}$. For the PBC simulations, Ewald summations were used to compute the electrostatic energy terms using the default parameters in the Sander module of the AMBER package ${ }^{26}$.

Trajectory analysis. Trajectory analysis was performed using CCPTRAJ from the AMBERTOOLS package ${ }^{30}$ or the homemade scripts developed in Tcl running in VMD 28 Calculations of RMSF per residue were performed against the average structure of the production runs in explicit solvent. SASA values for W90 were calculated for the entire 
production runs using a $1.4 \AA$ probe. The W90-Y88 reported distances were calculated as the distances between the centers of mass of the sidechains for both residues. For the SASA calculations, a spherical probe of $1.4 \AA$ radius/diameter was used. The SASA of the folded state was calculated from the average of the production run of the MD simulation of wild-type NS1 using VMD software ${ }^{28}$. For the calculation of the SASA in the unfolded state, an ensemble of 2000 configurations of the NS1 sequence encompassing residues 5 to 139 (corresponding to those present in the crystal structure ${ }^{12}$ was obtained using a flexible meccano's Monte Carlo algorithm ${ }^{31}$ using the ProtSA server ${ }^{28}$. Thus, the SASA for the unfolded state was calculated from the weighted average of the unfolded ensemble. The difference in solvent accessible surface area ( $\triangle S A S A)$ between the folded and unfolded states was calculated using the average SASA values of both states ${ }^{32}$.

\section{Unfolding Data Analysis.}

Unfolding Kinetics. The observed rate constants for unfolding reaction were obtained by fitting the kinetic traces to a bi-exponential function: 


$$
F(t)=\sum A_{n} \exp \left(-k_{n} t\right)+F_{\infty}
$$

where $(t)$ and $F_{\infty}$ are the observed signal at time $t$ and at infinite time, respectively, and An and $\mathrm{kn}$ are the signal amplitude and rate constants, respectively (for each process). The criteria followed to choose the minimal number of exponentials that provide the best fit for each trace were (1) a random dispersion of residuals, (2) low standard deviation values, and (3) significant improvement in the fit $\mathrm{X} 2$ values.

Equilibrium denaturation experiments. The $\mathrm{Gdm} . \mathrm{HCl}$ induced denaturation data were fitted to a two-state denaturation model that include linear slopes for the baselines, described in full in reference ${ }^{22}$. 


\section{RESULTS}

\section{Structural dynamics of wild-type NS1 and single proline mutants}

The crystal structure revealed a particular fold that does not appear as highly compact,

as revealed by the position of the C-terminal $\alpha 3$. To investigate the structural dynamics of NS1, we performed molecular dynamics (MD) simulations. We calculated the root mean squared fluctuation (RMSF) per residue and found that the most flexible regions correspond to the short loops $(11-19,52-54,60-68,82-83$, and $98-110)$ that flank the $\beta$ strands, and on a3 (Figure 1). The fluctuations of $\alpha 3$ arise from the concerted movement of the entire helix, which is displaced along its longitudinal axis in a back-and-forth movement along the surface of the core (not shown). 

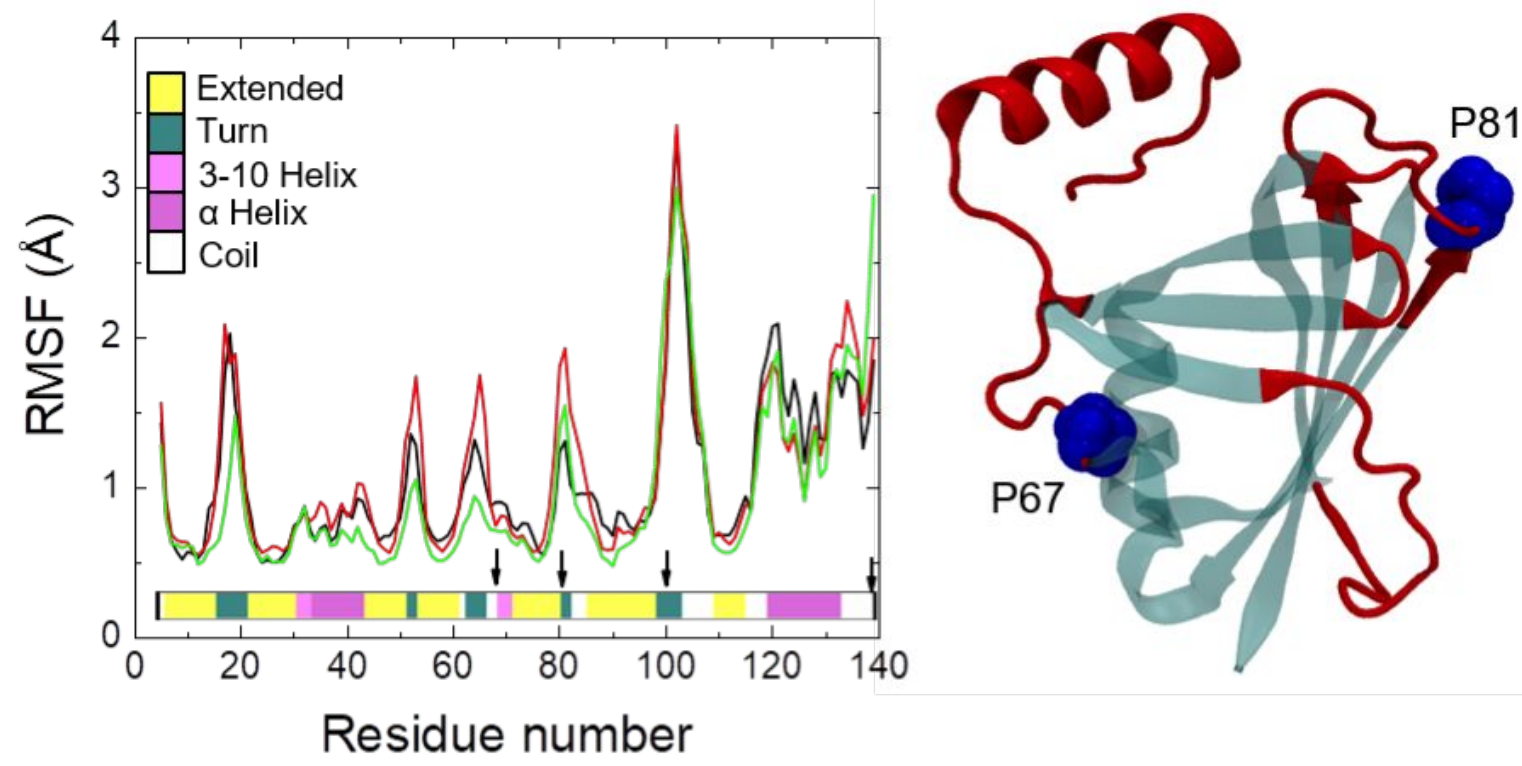

Figure 1. Structure and flexibility of NS1 WT and proline mutants. Left: The RMSF of WT and proline NS1 mutants was assessed analyzing the MD simulations obtained for WT NS1 (black), P67A (red) and P81A (green). The regions of higher motility correspond to loop and coil elements in the secondary structure (inset) and the $\alpha 3$ helix. Proline positions are indicated with arrows. Right: Crystal structure of WT NS1 obtained from PDB 5VJ2. The highly flexible regions are displayed in red, and the proline residues are shown in a VdW representation, with the conserved prolines P67 and P81 displayed as blue spheres.

Inspection of the secondary structure elements and RMSF show that the proline residues (P67, P81, P101 and $\mathrm{P} 139$ ) are located in regions of maximum mobility at the boundaries of secondary structural elements. However, P67 and P81 exhibit sequence conservation between human and bovine NS1 while P101 and P139 do not, where the latter is one residue away from the C-terminus. The role of the conserved prolines on the structure and dynamics of NS1 was addressed by generating the mutants P67A and P81A in silico. 
MD simulations reveal that these mutations do not impact on the protein fold substantially, but affect the dynamics both locally and non-locally. Both P67A and P81A exhibit an increased mobility of $\alpha 3$. The P67A variant exhibits increased a mobility in the 11-19 and $60-68$ loops. On the other hand, P81A exhibits a different behavior, where aside from the $\alpha 3$ helix, the rest of the protein displays a decreased RMSF, even in the flexible loops. Thus, although there is some local increase in flexibility in certain regions for this variant, the compact elements constituting the main $ß$-sheet fold of the core ${ }^{12}$ are more rigid.

\section{Effect of conserved prolines on the conformation and oligomerization of NS1}

Although the overall fold appears to be unmodified, as the 1D NMR spectra indicates

(Figure S2) there are some small changes on the CD spectra that can be pointed out. Mutation of P67 to alanine does not affect the secondary structure, as judged by an identical CD spectrum to wild type NS1 (Figure 2A). However, the single P81A mutant shows a significant secondary structure change, clearly evidenced in the difference spectrum (Figure $2 \mathrm{~A}$ inset). This change cannot be ascribed to a particular structural element, but only describe a change at around $225 \mathrm{~nm}$. 
P67A shows no difference from wild-type in terms of the tendency to form NS1SOs after temperature perturbation, as the oligomerization $T_{\text {m,app }}$ (Thermal transition midpoint) indicates, while P81A and the double mutant are more resistant to oligomerization with a 10 ${ }^{\circ} \mathrm{C}$ increase in the $\mathrm{T}_{\mathrm{m} \text {,app }}$ (Figure 2B, 2C and Table 1). Consistent with previous results ${ }^{16,17}$, the far UV CD spectra of the NS1SOs formed at the endpoint of the heating process shows an increase in ß-sheet content, and this is particularly noticeable for the P81A mutant (Figure $2 \mathrm{C}$ and Figure $\mathrm{S} 3$ ). 

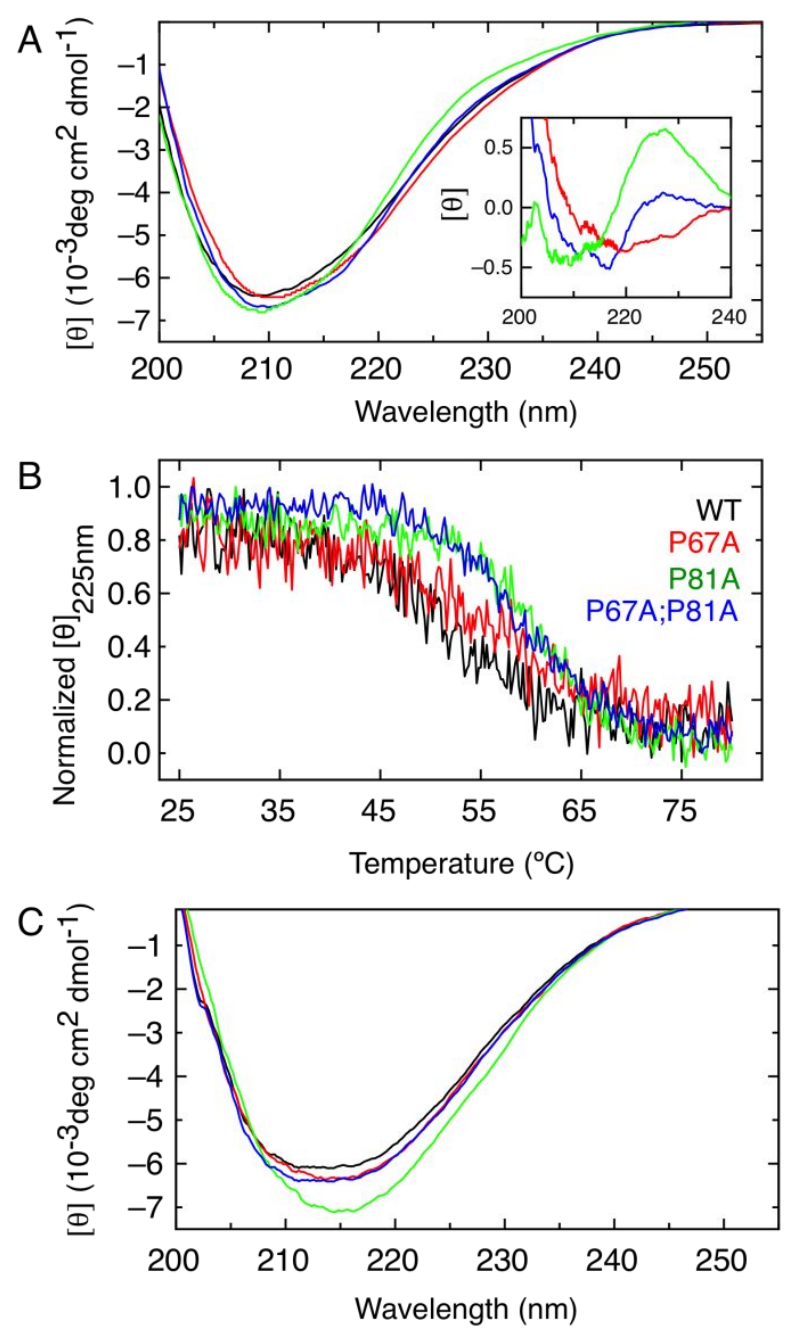

Figure 2. Secondary structure and heat-induced changes of WT NS1 and proline variants. (A) Far-UV CD spectra of WT NS1 (black), P67A (red), P81A (green) and P67A;P81A (blue) mutants at $25^{\circ} \mathrm{C}$; inset, difference spectra between wild-type and mutants (B). Normalized thermal scans of protein samples followed at $225 \mathrm{~nm}$ by far-UV CD. (C) CD spectra of wildtype and mutants at $80^{\circ} \mathrm{C}$ taken at the end of the thermal scan $(\mathrm{C})$. All the experiments were carried out in $10 \mathrm{mM}$ Tris. $\mathrm{HCl}(\mathrm{pH} \mathrm{8.0),} 100 \mathrm{mM} \mathrm{NaCl}$ and $1 \mathrm{mM}$ DTT at $10 \mu \mathrm{M}$ protein concentration. The same color code for the mutants is used in all figures. 
Table 1. Oligomerization and aggregation parameters ${ }^{\text {a }}$

\begin{tabular}{|c|c|c|c|c|}
\hline & \multirow{2}{*}{$\begin{array}{l}\mathrm{T}_{\mathrm{m}, \text { agg }} \\
\left({ }^{\circ} \mathrm{C}\right)\end{array}$} & \multirow{2}{*}{$\begin{array}{l}\mathrm{T}_{\mathrm{m} \text {, olig }} \\
\left({ }^{\circ} \mathrm{C}\right)\end{array}$} & \multicolumn{2}{|c|}{$k_{\mathrm{app} \times 10^{3}\left(\mathrm{~s}^{-1}\right)}$} \\
\hline & & & Fluo. ThT & Far UV-CD \\
\hline Wild-type & 65.2 & 46.9 & $1.2 \pm 0.003$ & $8.8 \pm 0.31$ \\
\hline P67A & 60.3 & 49.9 & $0.8 \pm 0.004$ & $10.4 \pm 0.39$ \\
\hline P81A & 67.6 & 56.7 & $0.5 \pm 0.001$ & $3 \pm 0.23$ \\
\hline P67A;P81A & 64.6 & 54.7 & $0.8 \pm 0.003$ & $6.7 \pm 0.22$ \\
\hline
\end{tabular}

aData obtained from figures 3 and 4 . Thermal scans were fit to an irreversible denaturation model 21,22 .

We have previously shown that the actual NS1SOs oligomerization process can be monitored by ThT binding which reports the formation of anti-parallel $ß$-sheet. This process is preceded by a marked secondary structure change evidenced by a decrease of ellipticity signal at $225 \mathrm{~nm}$ indicative of an increase in the $ß$-sheet content of the NS1 monomer ${ }^{16}$. We analyzed the oligomerization kinetics of the proline mutants by temperature jump (see materials and methods) and compared to the wild-type NS1 (Figure 3 and Figure S4). We found that P81A is significantly slower than wild-type, while P67A and the double mutant 
show similar kinetics, suggesting that P67 counterbalances the effect of P81. The nucleation lag is observed for all variants, but it is more pronounced in P81A. In any case, as we previously described, the oligomerization process is complex and multiple events may coexist within the $k_{\text {app }}$, which we now use to compare the global process.
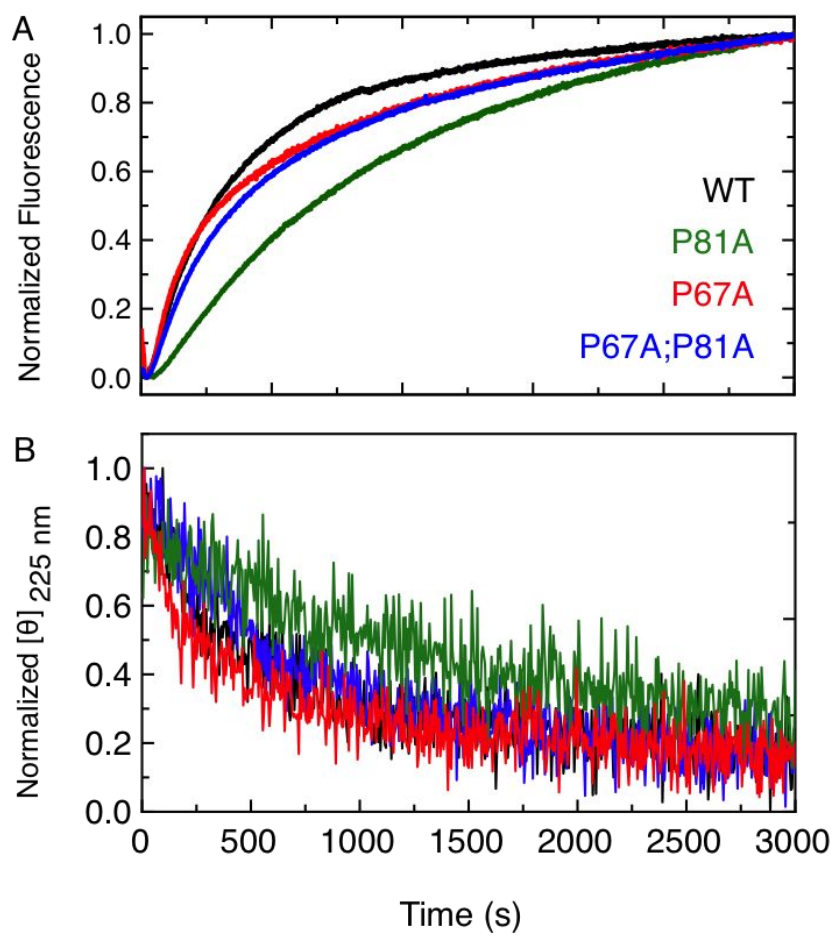

Figure 3. Kinetics of NS1SOs formation. Oligomerization kinetics were measured after transferring WT or NS1 mutants to a pre-equilibrated buffer at $47^{\circ} \mathrm{C}$ (Temperature jump) in four independent experiments. The final protein concentration was $10 \mu \mathrm{M}$. Oligomerization kinetics was assessed following changes in ThT fluorescence $(A)$ and in the far-UV CD signal at $225 \mathrm{~nm}$ (B). Traces were fitted to a single exponential function. The rate constants are shown in table 1. 
As in the case of WT, the kinetics of the secondary structure change of the mutants by $\mathrm{CD}$ precedes the oligomerization reaction as measured by ThT fluorescence, indicating an overall similar mechanism (Figure S4). Because the first-order nature of the conformational change, a single exponential fit yields a true constant $k_{\text {app }}$ P81A is substantially slower than WT while P67A shows a faster change. The double mutant shows an intermediate rate (Table 1).

\section{Competing soluble oligomerization and insoluble aggregation routes}

Partial protein losses during the purification process, possibly due to aggregation, prompted us to test the effect of ionic strength on the NS1 monomer. Increasing the concentration of $\mathrm{NaCl}$ does not affect the secondary structure at $25^{\circ} \mathrm{C}$ (not shown), but leads to aggregation upon increasing the temperature, as indicated by the complete loss of CD signal, increase in absorbance light scattering (not shown), and visual inspection after heating. We carried out thermal scanning experiments followed by CD and observed a drastic change in the scans from low to high salt (Figure 4A), where the salt effect on aggregation saturates at $0.2 \mathrm{M} \mathrm{NaCl}$ (Figure $4 \mathrm{~A}$, inset). The aspect of the scans clearly shows a shift from 
soluble oligomerization (NS1SOs species) at low salt characterized by a decrease in ellipticity due to the increase in $B$-sheet (Figure $2 B$ and Figure $4 A$ ), to the extreme insoluble aggregation, which we refer to as NS1Agg (Figure 4A). At intermediate salt concentrations, the opposed signal changes cancel out to different extents, indicating the presence of competing routes. Since there is no effect of the ionic strength on the CD of the NS1 monomer at $25^{\circ} \mathrm{C}$, we propose that the effect is after the formation of the oligomerization monomeric nucleus, i.e., during the oligomerization or aggregation processes.

A

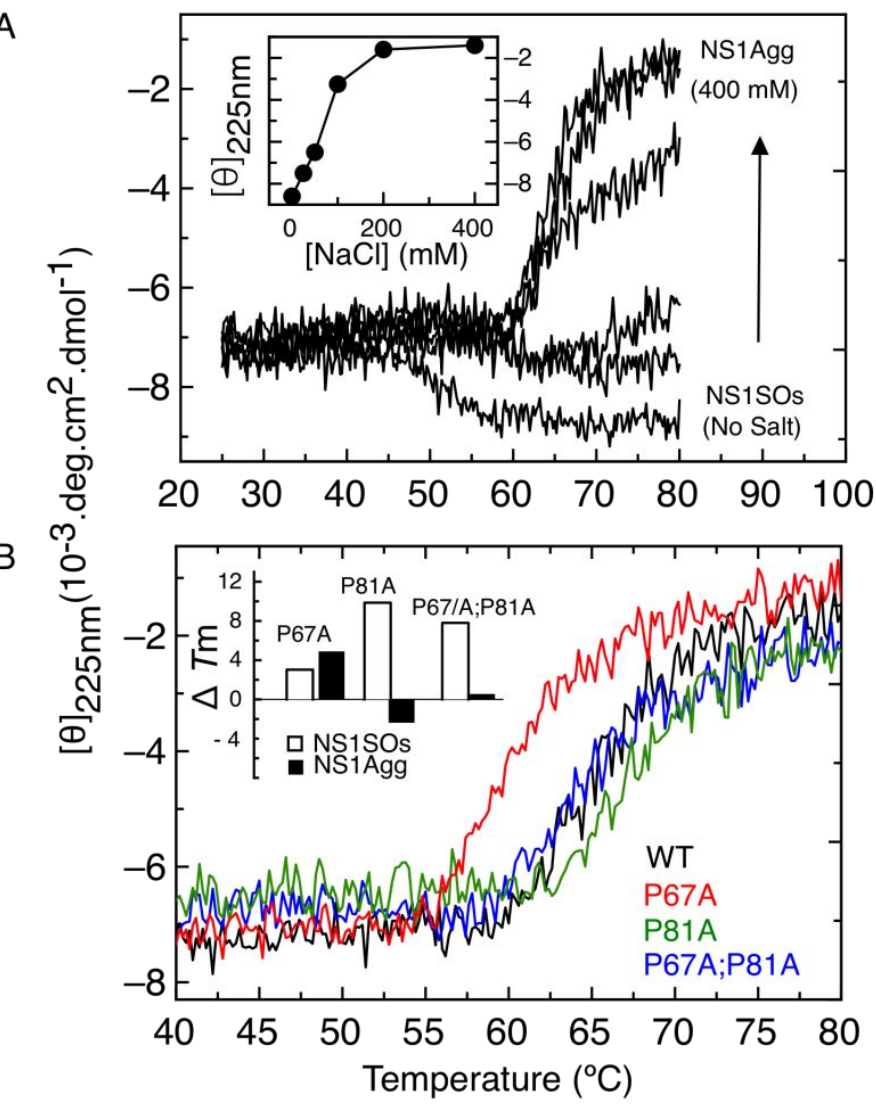

Figure 4. Effect of ionic strength in oligomerization and aggregation routes for WT NS1 and proline mutants. (A) Thermal scans, from $25^{\circ} \mathrm{C}$ to $80^{\circ} \mathrm{C}$ followed at $225 \mathrm{~nm}$ by far-UV CD, at 
different concentrations of $\mathrm{NaCl}$ for NS1 WT ([NaCl]: 0, 25, 50, 100, 200 and $400 \mathrm{mM}$ of $\mathrm{NaCl}$ ). Inset: dependence of the $\mathrm{CD}$ signal as function of the $\mathrm{NaCl}$ concentration at $80^{\circ} \mathrm{C}(\mathrm{B})$ Thermal scans of NS1 WT and mutants at $250 \mathrm{mM}$ of $\mathrm{NaCl}$ concentration followed by far-UV CD at $225 \mathrm{~nm}$. Inset: difference between the Tm obtained for mutants relative to WT NS1 protein. All experiments were performed at $10 \mu \mathrm{M}$ final protein concentration.

In order to test the effect of the proline mutants on the aggregation process, we carried

out thermal scans followed by CD. The P81A mutant is slightly but significantly more resistant to thermal aggregation when compared to wild-type, but P67A is substantially more aggregation prone, as a $5^{\circ} \mathrm{C}$ decrease in $\mathrm{T}_{\mathrm{m} \text {,app }}$ indicates (Figure 4B and Table 1).

\section{Folding of proline variants}

We have previously shown that NS1 unfolds reversibly and its chemical unfolding transition can be followed by its single buried tryptophan residue, an excellent reporter for global unfolding ${ }^{16}$. Upon progression of the unfolding process the exposure to the solvent, the maximum emission wavelength $\left(\lambda_{\max }\right)$ of the single Trp90 shifts from 332 to $356 \mathrm{~nm}$ (Figure S5). The process is also accompanied by a large change in fluorescence intensity $\left(F_{\text {int }}\right)$, likely because of quenching of its unique trp residue, since we use an excitation wavelength of $295 \mathrm{~nm}$ to eliminate possible contributions from tyrosine residues. 
The transition can also be also followed by far-UV circular dichroism which shows the disappearance of the signal corresponding to folded secondary structure, with maximum ellipticity change between 215 and $225 \mathrm{~nm}$ (Figure S5). Performing both measurements in the same experiment with a protein concentration that yields an optimal signal provides maximum accuracy for dissecting a complex transition. Based on both fluorescence and ellipticity transitions, we monitored the $F_{\text {int }}$ at $325 \mathrm{~nm}$ and the molar elipticity [ $\left.\theta\right]$ at $225 \mathrm{~nm}$. The ellipticity change upon unfolding of wild-type and mutants show a large pre-transition slope from zero to ca $2.5 \mathrm{M}$ denaturant, indicative of a substantial change of secondary structure, an anomalous signal of an aromatic residue, or both (Figure 5A). This linear transition is followed subsequently by a cooperative transition similar to what was previously observed ${ }^{16}$, whit a apparent similar behavior for the mutants. However, two salient features can be observed of this first transition: in P81A it takes place at higher denaturant than the other species and the P67A/P81A double mutant shows the largest change in secondary structure (Figure 5A).

When both $F_{\text {int }}$ and $[\theta]$ are represented for each species an evident uncoupling between the two signals is observed (Figure 5B), strongly suggesting a non two-state 
transition, with the largest uncoupling corresponding to P67A (Figure 5B, Figure S5). The partial overlapping of the two transitions, the absence of a significative $F_{\text {int }}$ change in the second transition, and the fact that the first secondary structure transition is large an opposed to the second more stable and cooperative one, makes fitting the data to a three-state model very difficult and therefore not quantitatively reliable. As an approximation, since the first ellipticity transition is linear, we fit it as a slope preceding the main cooperative/second transition treated as two-state in all four proteins and the data are presented in Table 2. Using the P67A as an example of the uncoupling between both signals (Figure 5B), we fitted the $\mathrm{F}_{\text {int }}$ to a two-state model in a range where the second transition has either not started or can be approximated as linear within the transition midpoint range. The resulting data are presented in Table 2.

Table 2. Thermodynamic parameters obtained by fitting the fluorescence or far-UV CD data to a two-state model.

\begin{tabular}{|c|c|c|c|c|c|c|}
\hline & \multicolumn{3}{|c|}{ First fluorescence transition } & \multicolumn{3}{|c|}{ Second Ellipticity transition } \\
\hline & $\begin{array}{c}\Delta G \\
\left(\mathrm{kcal} . \mathrm{mol}^{-1}\right)\end{array}$ & $\begin{array}{c}m \\
\left(\mathrm{kcal} . \mathrm{mol}^{-1} \cdot \mathrm{M}^{-1}\right)\end{array}$ & $D_{50 \%}$ & $\begin{array}{c}\Delta G \\
\left(\mathrm{kcal} . \mathrm{mol}^{-1}\right)\end{array}$ & $\begin{array}{c}m \\
\left(\mathrm{kcal} . \mathrm{mol}^{-1} \cdot \mathrm{M}^{-1}\right)\end{array}$ & $D_{50 \%}$ \\
\hline Wild-type & $3.21 \pm 0.51$ & $1.32 \pm 0.25$ & 2.4 & $3.50 \pm 0.3\{$ & $1.02 \pm 0.12$ & 3.4 \\
\hline P67A & $1.98 \pm 0.48$ & $1.29 \pm 0.19$ & 1.5 & $2.36 \pm 0.4$ & $0.69 \pm 0.15$ & 3.4 \\
\hline
\end{tabular}




\begin{tabular}{|c|c|c|c|c|c|c|}
\hline P81A & $5.03 \pm 0.76$ & $1.70 \pm 0.25$ & 2.9 & $5.88 \pm 0.7$ & $1.60 \pm 0.21$ & 3.6 \\
\hline$P 67 ; 81 A^{a}$ & nd & nd & $2.5^{b}$ & nd & nd & $3.3^{b}$ \\
\hline
\end{tabular}

The fact that the $m$ values are different suggest that there are differences in the exposure of solvent accessible surface area ( $\triangle A S A)$ across this first transition to an intermediate state, and renders the $\Delta G$ values not comparable (Table 2). Under these circumstances we use the denaturation midpoint $[D]_{50 \%}$, calculated from the $\Delta G$ and $m$ of each protein, as the best estimation for stability of this first transition. The P81A mutant is more stable than wild type, whereas P67A is less stable and the double mutant appears to compensate both single mutants, based on its apparent $[D]_{50 \%}$ of $2.5 \mathrm{M}($ Table 2$)$. These results are also visually confirmed when $\mathrm{F}_{\text {int }}$ is represented in a normalized plot (Figure $5 \mathrm{C}$ ).

Similarly, we approach the first secondary structure transition as a linear process and treat the second part of the curve as a two-state process. Once again, difference in $m$ values hampers the use of the $\Delta G$ for comparing the different proteins (Table 2). This allow us to conclude that the wild-type, P67A and P81A show similar stability in this cooperative transition, indicated by $[D]_{50 \%}$ between 3.4 and $3.6 \mathrm{M}$, which we ascribe to the folded $ß$-sheet core described in the X-ray structure ${ }^{12}$. The complexity of the double mutant is revealed by 
the partial reversibility as opposed to the complete secondary and tertiary structure reversibility for the single mutants and the wild-type protein (Figure S7). The partial reversibility preclude the extraction of accurate thermodynamic parameters. Irrespective of the degree of reversibility and its structural basis, we use the apparent $[D]_{50 \%}$ (3.3 M, Table 2 and Figure S6) for an approximation to stability. 


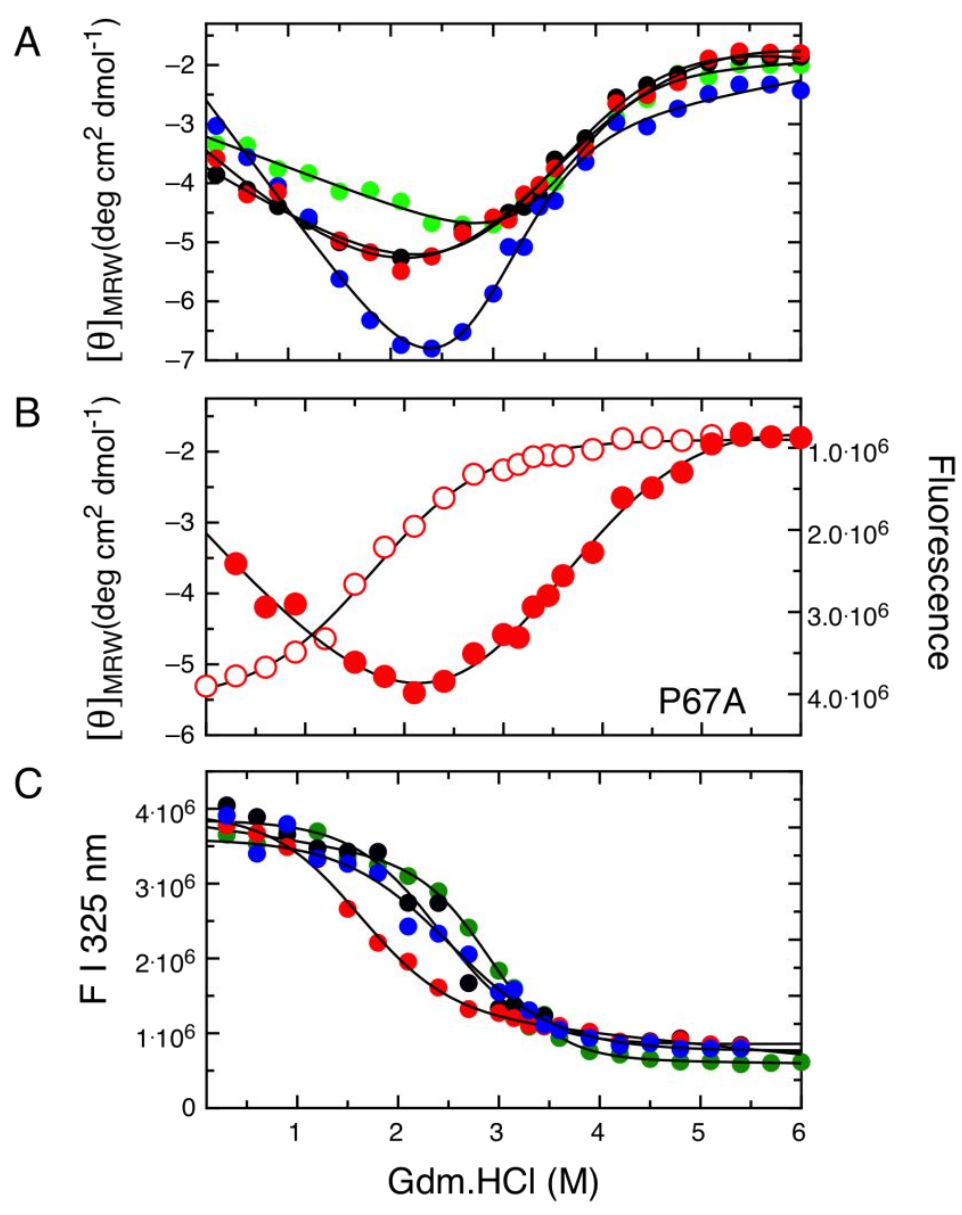

Figure 5. Gdm. $\mathrm{HCl}$ equilibrium denaturation of wild-type and proline mutants. (A) Denaturation followed by changes in molar ellipticity. (B) Denaturation followed by ellipticity at $225 \mathrm{~nm}$ and $F_{\text {int }}$ at $325 \mathrm{~nm}$ changes for the P67A mutant, shown as an example (see Figure S6). The fits to a two-state denaturation model are represented. (C) Comparison of $F_{\text {int }}$ of all proteins. All the measurements were carried out at $10 \mu \mathrm{m}$ protein concentration, and fluorescence and ellipticity data collected from the same experiments.

Given the impact of having very different $m$ values on overall stability, we tackled a kinetic analysis of the unfolding rates in order to obtain another assessment of stability. We have previously shown that unfolding shows a monoexponential decay with a minimal effect 
of the denaturant on the rate. We analyzed the unfolding rates $\left(k_{\mathrm{obs}}\right)$ of the mutants at $5.0 \mathrm{M}$ Gdm. $\mathrm{HCl}$ well after the completion of the unfolding transition, and compared them to the wildtype protein (Figure 5C, Figure S5 and Table S1). All proteins showed a single phase, but a faster fluorescence change cannot be determined because of the experimental dead time of manual mixing. P81A unfolds slightly slower than wild type but P67A unfolds 10-fold faster (Figure 6A). The double mutant also unfolds 10 -fold faster, which indicates that the unfolding rate is governed by P67, different from the effect at the equilibrium. These results suggest an effect at the level of the transition state ensemble that requires further investigation. In the second transition detected in the three mutants, secondary and tertiary structure unfold together similar to wild-type (Figure $6 \mathrm{~B}$ and Table 2), where only a single phase can be detected because of $C D$ noise and also the experimental deadtime of manual mixing. 


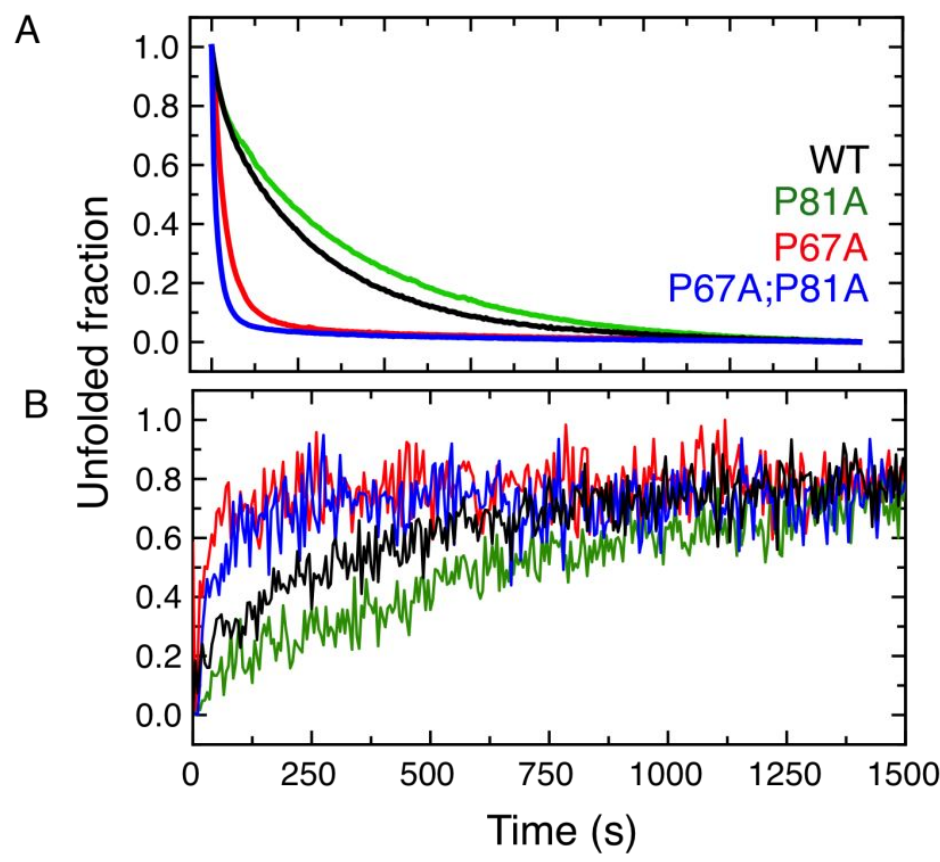

Figure 6. Gdm.HCl unfolding of wild-type and mutant NS1s. Each protein was added to a cuvette containing $5.0 \mathrm{M} \mathrm{Gdm} . \mathrm{HCl}$ in buffer and either fluorescence $(A)$ or ellipticity $(B)$ was followed in similar conditions to equilibrium unfolding experiments (Figure 5).

Fluorescence changes reveal local dynamics at the C-term helix/ß-sheet core interface.

We have shown that the fluorescence intensity at the $\lambda_{\max }(360 \mathrm{~nm})$ shows a marked change prior to the global unfolding transition reported by changes in $\lambda_{\max }$ with a differential effect depending on the mutant, reflecting a highly sensitive reporter for local events (Figure 5B, Figure S5). In order to understand the basis for this phenomenon, we carried out MD simulation analysis of the single tryptophan 90 . Although the calculations of the SASA on the 
crystal structure of NS1 yields this Trp side chain as buried, trajectory analysis reveal that the exposure of the residue oscillates, from 0 to ca $26 \AA^{2}$ in WT NS1. This large fluctuation shows Trp90 as partly exposed, and the level of exposure correlates with the position of $\alpha-$ helix 3 along the trajectory, as in some arrangements it completely occludes the Trp side chain. P67A follows a similar pattern to that of WT NS1, although with a slightly higher mean and a broader distribution (Figure 7B). P81A clearly shows both a lower mean accessibility and less variability, correlating with an overall lower flexibility of the protein as assessed by RMSF calculations (Figure 1).
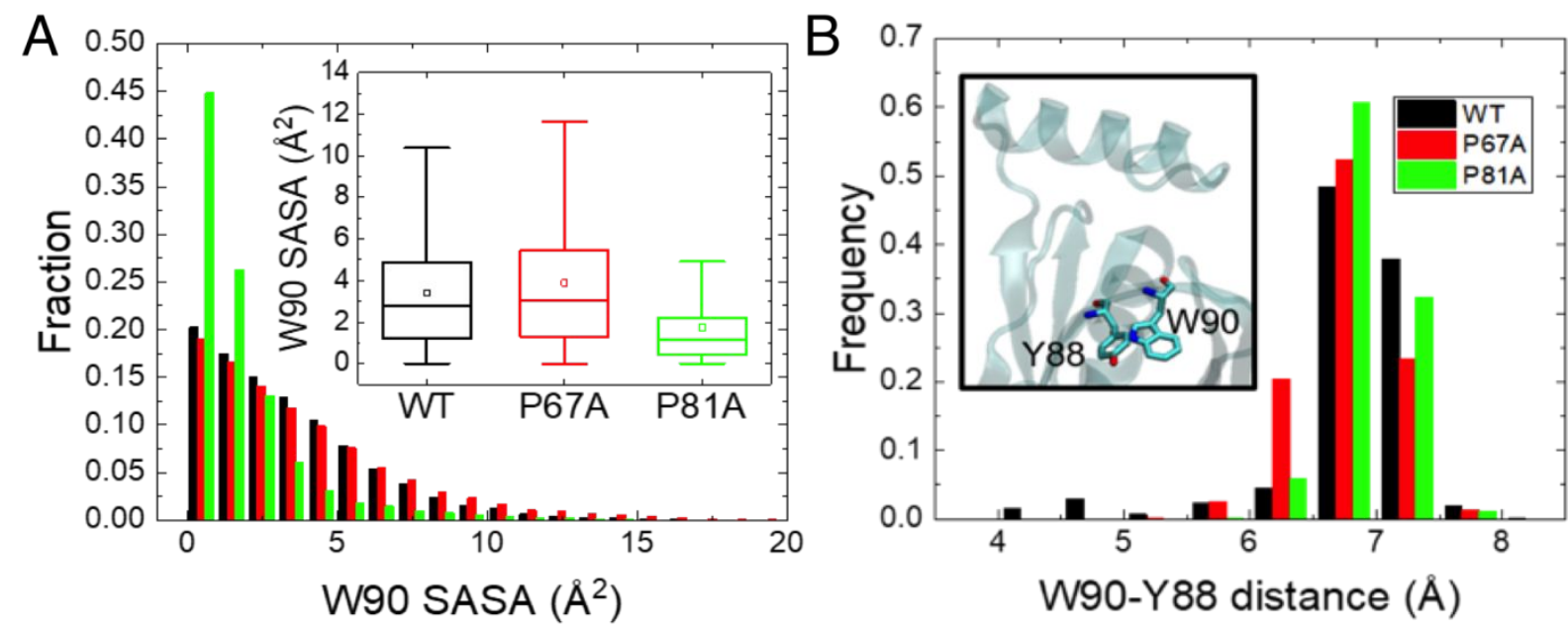

Figure 7. Solvent accessibility for WT and NS1 proline mutants by MD dynamics. (A) Solvent accessibility of W90 in NS1 variants. Frequency of the solvent accessible surface area (SASA) distribution was obtained calculating the SASA of W90, along the 100ns trajectories, for WT NS1 (black), P67A (red) and P81A (green). Inset: Box-and-whiskers plot, showing the mean, median, interquartile range (IQR) Q3-Q1 that represents the data range between $25 \%$ 
and $75 \%$ of the data, and data range within 1.5 IQR calculated as Q1-1.5IQR to Q3+1.5IQR. (B) Distance distribution between W90 and Y88 in NS1 variants. The distances along the trajectories were calculated between the center of mass of the sidechains of residues W90 and Y88 (inset). The frequency of the distances distribution is plotted using the calculations performed along the 100ns trajectories for WT NS1 (black), P67A (green) and P81A (red)

Another factor that can affect fluorescence is quenching, whether collisional or due to

Förster Resonance energy transfer (FRET). Visual inspection of NS1 structure shows that tyrosine 88 side chain lies on top of Trp90, rendering it a suitable candidate as the fluorescence quencher (Figure 7B). We analyzed the distance between both residues, calculating the distance between the respective centers of mass of the side chains along the dynamics for WT NS1 and both mutants. The displacement of $\alpha$-helix along the trajectories for WT NS1 modulates the Trp90-Tyr88 distance distribution, showing a bimodal distribution with the maxima centered around $7 \AA$ and $4.5 \AA$, respectively, where the latter distribution being much less populated. Both mutants display the same trend, evidenced in the RMSF and SASA calculations. They also display Trp90-Tyr88 distance distribution centered around $7 \AA$, with P67A exhibiting a broader distribution, favoring closer distances while P81A presents a narrower distribution skewed towards larger distances. 


\section{DISCUSSION}

In previous work we showed that NS1 from human RSV, although a stable monomer in solution, shows a marked tendency to form soluble, stable and regular spherical oligomers at physiological temperatures, which we termed NS1SOs ${ }^{16}$. This process involves a slow conformational change corresponding to an increased ß-sheet content prior to oligomerization. We hypothesized that such change may originate in conformational isomerizations from two highly conserved proline residues and at the same time gain more insight into the mechanism. The recently determined crystallographic structure provides structural grounds for interpretation of our current and previous results.

The experimental results and MD simulations show that the overall structure is not affected by the proline to alanine substitutions. However, the calculations expose some important details of the protein dynamics and the role of the conserved prolines. These mutations elicit both local and non-local effects on the protein dynamics, that in turn affect overall protein stability and more importantly soluble and insoluble aggregation routes. MD simulations show several flexible ß-strand flanking loops which become rigid upon mutation of proline 81 to alanine, particularly in the $60-68$ loop. Interestingly, a digitization observed for 
most regions strongly suggests a global structural impact of this proline residue. MD also reveals a fluctuation of its $\mathrm{C}$-terminal $\alpha 3$ helix which displaces along the main $ß$-strand barreltype core ${ }^{12}$.

In experimental terms, the P81A mutant shows a significant increase in ellipticity at 225 $\mathrm{nm}$, although the 1D, although no large differences are observed in 1D-NMR. This mutant also leads to a decrease in oligomerization propensity as indicated by an increased $T_{m \text {,app. }}$. Both the CD of the monomer and decreased tendency to oligomerization by temperature are identical for P81A and the double mutant P67A;P81A, strongly suggesting that the effect at the equilibrium is governed by $\mathrm{P} 81$. The oligomerization rate of P81A is also slower than the WT and the other variants for both temperature induced conformational change and the subsequent oligomerization reaction. We propose that the slower conformational change responds to the rigidization and stabilization caused by P81 on the overall structure resulting in a higher energy barrier. The protein is a monomer at low concentrations in solution, but the oligomerization mechanism and the crystallographic structure support the existence of a dimeric species which we believe is the immediate product of the conformational change required for oligomerization to take place ${ }^{12,17}$. An interesting observation is that P67A reverts 
the effect of P81 on the oligomerization kinetics but not on the equilibrium temperature transition suggesting a differential role in ground and transition states.

In this work, we uncover insoluble aggregation pathway (NS1Agg) that takes place at increasing salt concentration which competes with the NS1SOs soluble oligomerization route peaking at $0.2 \mathrm{M} \mathrm{NaCl}$. This process is marginally affected by $\mathrm{P} 81$, but strongly increased by P67, suggesting that both soluble oligomerization and insoluble aggregation routes respond to different intermediate conformers, supported by the lack of correlation of the effect of mutation on both processes (Figure 4B, inset). It also suggests that depending on its concentration levels in the host cell and its ligands, it may be a preferred pathway for unbound NS1, a possible scenario of high levels of the protein ${ }^{8}$. In any case, the fact that the ionic strength favors the aggregation route (NS1Agg), suggests a very sensitive electrostatic component on the soluble oligomerization NS1SOs route screened by the addition of $0.2 \mathrm{M}$ salt. This explains the apparent contradiction observed in the lack of effect of residues F17, F56, Y125 in which structure clearly links them to the stabilization of the C-terminal helix, since the thermal denaturation reported therein is in fact insoluble aggregation ${ }^{12}$. 
Chemical denaturation experiments show a first weaker transition with a large change

in fluorescence intensity, but an otherwise small change in $\lambda_{\max }$, and a second transition where the $\lambda_{\max }$ change is that expected from a fully unfolded tryptophan. Circular dichroism also shows two marked transitions, strongly suggesting that the first transition is of significant structural magnitude. However, we believe that anomalous contribution of aromatics to the ellipticity signal in that wavelength range plays an important role. P81A shows the most stabilizing effect on this transition, P67A is destabilizing compared to wild-type, and the double mutant appears to compensate the individual single mutations. Neither of the mutants appear to significantly affect the second stronger and more cooperative transition. We propose that this first weak transition corresponds to unfolding of the alpha-helix-3 ${ }^{12}$, which leaves an intermediate species corresponding to the main folding ß-sheet core. In the absence of denaturant this species is not soluble as a monomer and oligomerizes as the temperature jump experiments indicate. Both P81 and P67 residue plays opposing but otherwise similar roles in chemical partial unfolding and temperature stabilization.

We have previously shown that, as opposed to the single phase unfolding reaction, the refolding kinetics is a complex multiphasic reaction based on oligomerization of its proline 
residues prior to the global refolding ${ }^{17}$. This implies that elements of structure in the unfolded state must isomerize for the refolding to take place, and this isomerization would modulate the formation or disruption of a folding nucleus. P67 exists as the trans isomer in the folded state, and its replacement by alanine appears to favor the formation of local structure. The local residual structure is probed by a strong quenching of Trp90, supporting the hypothesis. On the other hand, P81 populates the cis isomer in the native folded state, and its mutation favors rigidization and opposes to oligomerization. The absence of the WT P81 would preclude an isomerization dependent conformational change, but further interpretation requires NMR analysis.

The overall picture shows a weak and highly mobile C-terminal $\alpha$-helix that is readily affected by mild temperature perturbation that leads to either oligomerization or aggregation routes depending on the ionic strength. This helix weakens upon chemical unfolding, as reported by its single and highly sensitive Trp90, preceding the global unfolding transition corresponding to the main $ß$-sheet core ${ }^{12}$. Although there is no specific protein target defined in therms of binding affinity or structure, this mobile C-terminal helix might have important implications in structure ${ }^{33}$. The conserved proline residues, in particular P81, maintain the 
global structure dynamic in order for the oligomerization to be modulated. Thus, they appear to tune the exposure of SASA in order to either bind to the many interaction partners reported so far, or partake in those interactions involving oligomerization. Further functional analysis with the infection model aiming at the role of the oligomerization routes and interaction partners should be addressed by probing the C-terminal helix with biophysical and structural methods. The final goal is understanding the molecular basis of its interferon inhibiting activity in concerted action with NS2, both unique to RSV, particularly through the numerous interaction partners identified by proteomic studies, where oligomerization appears as a prominent feature for the biological activity this protein. 
Supporting Information Available: Supplementary figures and table. This material is available free of charge via the Internet at http://pubs.acs.org.

\section{Corresponding Author}

* corresponding author: gpg@leloir.org.ar. Phone: + 5411 52387500, ext. 3209. Fax: +5411

52387501

\section{Present Addresses}


Leonardo G. Alonso, Instituto de Nanobiotecnología (NANOBIOTEC), CONICET-Universidad de Buenos Aires, Junín 956, 1113 CABA, Argentina.

Damian A/varez-Paggi, INFANT Foundation, CONICET, Gavilan 94, CABA, Argentina

\section{Funding sources}

This work was supported by Agencia Nacional de Promoción Científica y Tecnológica

(ANPCyT, PICT 2015-1135). JC holds a PhD fellowship from CONICET; DAP, SAE, SSB,

MA, LGA and GPG are career investigators from CONICET.

\section{Access to ID}

NS1 X5FN71 


\section{REFERENCES}

(1) Gotoh, B., Komatsu, T., Takeuchi, K., and Yokoo, J. (2001) Paramyxovirus accessory proteins as interferon antagonists. Microbiol. Immunol. 45, 787-800.

(2) Parks, G. D., and Alexander-Miller, M. A. (2013) Paramyxovirus Activation and Inhibition of Innate Immune Responses. J. Mol. Biol. 425, 4872-4892.

(3) Spann, K. M., Tran, K.-C., Chi, B., Rabin, R. L., and Collins, P. L. (2004) Suppression of the induction of alpha, beta, and lambda interferons by the NS1 and NS2 proteins of human respiratory syncytial virus in human epithelial cells and macrophages [corrected]. J. Virol. 78, 4363-4369. (4) Swedan, S., Musiyenko, A., and Barik, S. (2009) Respiratory Syncytial Virus Nonstructural Proteins Decrease Levels of Multiple Members of the Cellular Interferon Pathways. J. Virol. 83, 96829693.

(5) Afonso, C. L., Amarasinghe, G. K., Bányai, K., Bào, Y., Basler, C. F., Bavari, S., Bejerman, N., Blasdell, K. R., Briand, F.-X., Briese, T., Bukreyev, A., Calisher, C. H., Chandran, K., Chéng, J., Clawson, A. N., Collins, P. L., Dietzgen, R. G., Dolnik, O., Domier, L. L., Dürrwald, R., Dye, J. M., Easton, A. J., Ebihara, H., Farkas, S. L., Freitas-Astúa, J., Formenty, P., Fouchier, R. A. M., Fù, Y., Ghedin, E., Goodin, M. M., Hewson, R., Horie, M., Hyndman, T. H., Jiāng, D., Kitajima, E. W., Kobinger, G. P., Kondo, H., Kurath, G., Lamb, R. A., Lenardon, S., Leroy, E. M., Li, C.-X., Lin, X.-D., Liú, L., Longdon, B., Marton, S., Maisner, A., Mühlberger, E., Netesov, S. V., Nowotny, N., Patterson, J. L., Payne, S. L., Paweska, J. T., Randall, R. E., Rima, B. K., Rota, P., Rubbenstroth, D., Schwemmle, M., Shi, M., Smither, S. J., Stenglein, M. D., Stone, D. M., Takada, A., Terregino, C., Tesh, R. B., Tian, J.-H., Tomonaga, K., Tordo, N., Towner, J. S., Vasilakis, N., Verbeek, M., Volchkov, V. E., Wahl-Jensen, V., Walsh, J. A., Walker, P. J., Wang, D., Wang, L.-F., Wetzel, T., Whitfield, A. E., Xiè, J., Yuen, K.-Y., Zhang, Y.-Z., and Kuhn, J. H. (2016) Taxonomy of the order Mononegavirales: update 2016. Arch. Virol. 161, 2351-2360.

(6) Lamb, R.A., and Parks, G.D. (2007) Paramyxoviridae: The viruses and Their Replication, in Fields' Virology David Mahan Knipe, Peter M. Howley. Lippincott Williams \& Wilkins, Philadelphia.

(7) Collins, P. L., and Crowe, J. E. (2007) Respiratory Syncytial virus and metapneumovirus, in Fields' Virology David Mahan Knipe, Peter M. Howley. Lippincott Williams \& Wilkins, Philadelphia.

(8) Fearns, R., and Collins, P. L. (1999) Role of the M2-1 transcription antitermination protein of respiratory syncytial virus in sequential transcription. J. Virol. 73, 5852-5864.

(9) Collins, P. L. (2011) Human respiratory syncytial virus Siba K. Samal. Caister Academic Press, Norfolk, UK.

(10) Atreya, P. L., Peeples, M. E., and Collins, P. L. (1998) The NS1 protein of human respiratory syncytial virus is a potent inhibitor of minigenome transcription and RNA replication. J. Virol. 72, 1452-1461.

(11) Wu, W., Tran, K. C., Teng, M. N., Heesom, K. J., Matthews, D. A., Barr, J. N., and Hiscox, J. A. (2012) The Interactome of the Human Respiratory Syncytial Virus NS1 Protein Highlights Multiple Effects on Host Cell Biology. J. Virol. 86, 7777-7789.

(12) Chatterjee, S., Luthra, P., Esaulova, E., Agapov, E., Yen, B. C., Borek, D. M., Edwards, M. R., Mittal, A., Jordan, D. S., Ramanan, P., Moore, M. L., Pappu, R. V., Holtzman, M. J., Artyomov, M. N., Basler, C. F., Amarasinghe, G. K., and Leung, D. W. (2017) Structural basis for human respiratory syncytial virus NS1-mediated modulation of host responses. Nat. Microbiol. 2, 17101.

(13) Boyapalle, S., Wong, T., Garay, J., Teng, M., San Juan-Vergara, H., Mohapatra, S., and Mohapatra, S. (2012) Respiratory syncytial virus NS1 protein colocalizes with mitochondrial antiviral signaling protein MAVS following infection. PloS One 7, e29386.

(14) Ren, J., Liu, T., Pang, L., Li, K., Garofalo, R. P., Casola, A., and Bao, X. (2011) A novel 
mechanism for the inhibition of interferon regulatory factor-3-dependent gene expression by human respiratory syncytial virus NS1 protein. J. Gen. Virol. 92, 2153-2159.

(15) Ling, Z., Tran, K. C., Arnold, J. J., and Teng, M. N. (2008) Purification and characterization of recombinant human respiratory syncytial virus nonstructural protein NS1. Protein Expr. Purif. 57, 261270.

(16) Pretel, E., Camporeale, G., and de Prat-Gay, G. (2013) The Non-Structural NS1 Protein Unique to Respiratory Syncytial Virus: A Two-State Folding Monomer in Quasi-Equilibrium with a Stable Spherical Oligomer. PLoS ONE (Pastore, A., Ed.) 8, e74338.

(17) Pretel, E., Sánchez, I. E., Fassolari, M., Chemes, L. B., and de Prat-Gay, G. (2015) Conformational Heterogeneity Determined by Folding and Oligomer Assembly Routes of the Interferon Response Inhibitor NS1 Protein, Unique to Human Respiratory Syncytial Virus. Biochemistry 54, 5136-5146.

(18) Swedan, S., Andrews, J., Majumdar, T., Musiyenko, A., and Barik, S. (2011) Multiple functional domains and complexes of the two nonstructural proteins of human respiratory syncytial virus contribute to interferon suppression and cellular location. J. Virol. 85, 10090-10100.

(19) Goswami, R., Majumdar, T., Dhar, J., Chattopadhyay, S., Bandyopadhyay, S. K., Verbovetskaya, V., Sen, G. C., and Barik, S. (2013) Viral degradasome hijacks mitochondria to suppress innate immunity. Cell Res. 23, 1025-1042.

(20) Taler-Verčič, A., Hasanbašić, S., Berbić, S., Stoka, V., Turk, D., and Žerovnik, E. (2017) Proline Residues as Switches in Conformational Changes Leading to Amyloid Fibril Formation. Int. J. Mol. Sci. $18,549$.

(21) Sanchez-Ruiz, J. M. (1992) Theoretical analysis of Lumry-Eyring models in differential scanning calorimetry. Biophys. J. 61, 921-935.

(22) Chemes, L. B., Noval, M. G., Sánchez, I. E., and de Prat-Gay, G. (2013) Folding of a cyclin box: linking multitarget binding to marginal stability, oligomerization, and aggregation of the retinoblastoma tumor suppressor AB pocket domain. J. Biol. Chem. 288, 18923-18938.

(23) P. Plateau, M. G. (1984) Exchangeable Proton NMR without Base-Line Distortion, Using New Strong-Pulse Sequences. J. Am. Chem. Soc. 104, 7310-7311.

(24) Delaglio, F., Grzesiek, S., Vuister, G. W., Zhu, G., Pfeifer, J., and Bax, A. (1995) NMRPipe: a multidimensional spectral processing system based on UNIX pipes. J. Biomol. NMR 6, 277-293.

(25) Schymkowitz, J., Borg, J., Stricher, F., Nys, R., Rousseau, F., and Serrano, L. (2005) The FoldX web server: an online force field. Nucleic Acids Res. 33, W382-W388.

(26) Salomon-Ferrer, R., Case, D. A., and Walker, R. C. (2013) An overview of the Amber biomolecular simulation package: Amber biomolecular simulation package. Wiley Interdiscip. Rev. Comput. Mol. Sci. 3, 198-210.

(27) Maier, J. A., Martinez, C., Kasavajhala, K., Wickstrom, L., Hauser, K. E., and Simmerling, C. (2015) ff14SB: Improving the Accuracy of Protein Side Chain and Backbone Parameters from ff99SB. J. Chem. Theory Comput. 11, 3696-3713.

(28) Humphrey, W., Dalke, A., and Schulten, K. (1996) VMD: Visual molecular dynamics. J. Mol. Graph. 14, 33-38.

(29) Berendsen, H. J. C., Postma, J. P. M., van Gunsteren, W. F., DiNola, A., and Haak, J. R. (1984) Molecular dynamics with coupling to an external bath. J. Chem. Phys. 81, 3684-3690.

(30) Roe, D. R., and Cheatham, T. E. (2013) PTRAJ and CPPTRAJ: Software for Processing and Analysis of Molecular Dynamics Trajectory Data. J. Chem. Theory Comput. 9, 3084-3095.

(31) Lovell, S. C., Davis, I. W., Arendall, W. B., de Bakker, P. I. W., Word, J. M., Prisant, M. G., Richardson, J. S., and Richardson, D. C. (2003) Structure validation by C $\alpha$ geometry: $\phi, \psi$ and C $\beta$ deviation. Proteins Struct. Funct. Bioinforma. 50, 437-450.

(32) Bernadó, P., Blackledge, M., and Sancho, J. (2006) Sequence-Specific Solvent Accessibilities of Protein Residues in Unfolded Protein Ensembles. Biophys. J. 91, 4536-4543. 
(33) Tremiño, L., Forcada-Nadal, A., Contreras, A., and Rubio, V. (2017) Studies on cyanobacterial protein PipY shed light on structure, potential functions, and vitamin B6 -dependent epilepsy. FEBS Lett. 591, 3431-3442. 


\title{
For Table of Contents Use Only
}

\section{Conformational isomerization involving conserved proline} residues modulates oligomerization of the NS1 interferon response inhibitor from the syncytial respiratory virus

\author{
Julieta Conci', Damian Alvarez-Paggi', Guilherme A. P. de Oliveira², Talita D. Pagani, \\ Sebastian A. Esperante1, Silvina S. Borkosky¹, Martin Aran'1, Leonardo G. Alonso', Ronaldo \\ Mohana-Borges ${ }^{3}$, Gonzalo de Prat-Gay ${ }^{1 *}$.
}




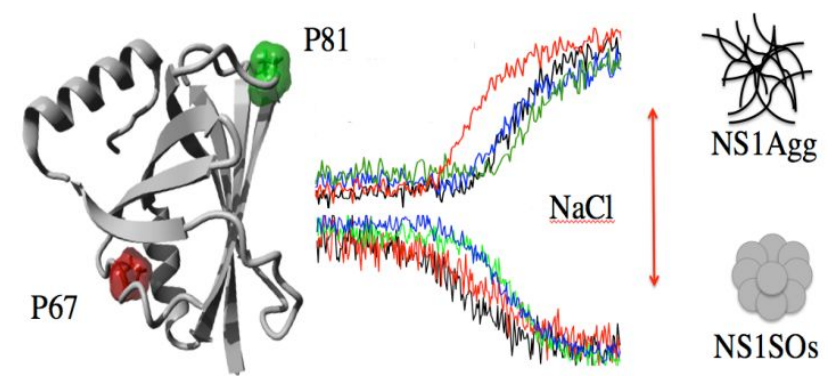

